\title{
Effect of nanocellulose isolation techniques on the formation of reinforced poly(vinyl alcohol) nanocomposite films
}

\author{
Y. M. Zhou', S. Y. Fu' ${ }^{1 *}$ L. M. Zheng ${ }^{2}$, H. Y. Zhan ${ }^{1}$ \\ ${ }^{1}$ State Key Laboratory of Pulp and Paper Engineering, College of Light Industry and Food Science, South China \\ University of Technology, Guangzhou, 510640 Guangdong, China \\ ${ }^{2}$ School of Chemistry and Chemical Engineering, South China University of Technology, Guangzhou, 510640 \\ Guangdong, China
}

Received 19 February 2012; accepted in revised form 1 May 2012

\begin{abstract}
Three techniques including acid hydrolysis (AH), 2,2,6,6-tetramethylpiperidine-1-oxyl radical (TEMPO)-mediated oxidation (TMO) and ultrasonication (US) were introduced to isolate nanocellulose from microcrystalline cellulose, in order to reinforce poly(vinyl alcohol) (PVA) films. Important differences were noticed in fiber quality of nanocellulose and film properties of PVA nanocomposite films. The TMO treatment was more efficient in nanocellulose isolation with higher aspect ratio, surface charge $(-47 \mathrm{mV})$ and yields $(37 \%)$. While AH treatment resulted in higher crystallinity index $(88.1 \%)$ and better size dispersion. The fracture surface, thermal behavior and mechanical properties of the PVA nanocomposite films were investigated by means of scanning electron microscopy (SEM), differential scanning calorimetry (DSC), thermogravimetric analysis (TGA) and tensile testing. The results showed that both the TMO-derived and AH-derived nanocellulose could be dispersed homogeneously in the PVA matrices. AH/PVA films had higher elongation at break $(51.59 \%$ at $6 \mathrm{wt} \%$ nanocellulose loading) as compared with TMO/PVA, while TMO/PVA films shown superior tensile modulus and strength with increments of $21.5 \%$ and $10.2 \%$ at $6 \mathrm{wt} \%$ nanocellulose loading. The thermal behavior of the PVA nanocomposite films was higher improved with TMO-derived nanofibrils addition.
\end{abstract}

Keywords: nanocomposites, isolation techniques, cellulose, reinforcements

\section{Introduction}

Owing to environmentally friendly attributes, good mechanical properties, low density, biodegradability and abundant availability of renewable resources, the production of nanocellulose and their application in composites materials has gained increasing attention in recent times [1-2]. Nanocelluloses are recognized to be more effective in reinforcing polymers, due to the interaction between the nano-sized elements that form a percolated network connected by hydrogen bonding [3]. Two different types of nanocellulose can be isolated from a cellulosic source: nanocrystals and nanofibrils. Nanocrystals have a perfect crystalline structure and high modu- lus, close to the theoretical modulus of cellulose; nanofibrils are fibrillar units containing both amorphous and crystalline regions and have the ability to create entangled networks [4]. Different properties of these two types of nanocellulose will result in varying reinforcement of nanocomposites.

In order to utilize nanocellulose as a reinforcing phase to form nanocomposites, the strong hydrogen bonding between cellulose crystals must be separated and dispersed well in the polymer matrices [5]. Extensive research has been reported to extract nanocellulose from different sources [6-10]. Typical processes involve mechanical and chemical treatments. The chemical ways, mainly by strong

\footnotetext{
${ }^{*}$ Corresponding author, e-mail: shyfu@scut.edu.cn
}

(c) BME-PT 
acid hydrolysis, can remove the amorphous regions of cellulose fibers and produce cellulose nanocrystals [11-12]. While for mechanical methods, which include high intensity ultrasonication [13], high pressure refiner [14] or grinder treatment [15], the main product generated is not a single fiber and has been referred as nanofibrils. However, these two techniques of extracting nanocellulose from plants are time consuming and very costly [1]. It involves high consumption of energy for processes as mechanical treatments [16], which can cause dramatic decrease in both the yield and fibril length down to $100-150 \mathrm{~nm}$ and also introduces damage to the environment, as in the case of chemical treatments [17]. Current research has been focused on finding environmental conservation, high efficiency and low costs methods to isolate nanocellulose. Recently, individualized cellulose nanofibrils have been obtained using 2,2,6,6-tetramethylpiperidine-1-oxyl radical (TEMPO)-mediated oxidation for regioselective conversion of the cellulose primary hydroxyl groups to aldehydes and carboxylate ones. The mild reaction condition (room temperature and alkalescent medium), the characteristic of little fiber morphological change and the resultant diverse surface functionalities (carboxyl, aldehyde, and hydroxyl) lend the TEMPO-mediated oxidation technique significant potential in the fields of composites reinforcement [18-19].

Currently, very few references are available about the systematic study of the effect of nanocellulose isolation techniques on the quality of nanocellulose and its performance in reinforced nanocomposites. The main goal of this work is to employ three different techniques including acid hydrolysis $(\mathrm{AH})$, TEMPO-mediated Oxidation (TMO) and ultrasonication (US) to isolate nanocellulose from microcrystalline cellulose (MCC) and to evaluate the quality of nanocellulose and the reinforcing ability of these nanocellulose in PVA matrices.

\section{Experiment}

\subsection{Materials}

Microcrystalline cellulose (MCC) with a mean diameter size of $20 \mu \mathrm{m}$, purchased from Xuanyuan Machinery (Shandong, China) was used as raw material. Poly(vinyl alcohol) (PVA, 99\% hydrolyzed, $M_{\mathrm{w}} 85000 \sim 124000$ ) was used as matrices. Sulfuric acid $(98 \mathrm{wt} \%)$ was used for the acid hydrolysis of
MCC. 2,2,6,6-tetramethylpiperidine-1-oxyl radical (TEMPO), sodium bromide $(\mathrm{NaBr})$ and $6 \%$ sodium hypochlorite $(\mathrm{NaClO})$ solution were used for the TEMPO-mediated oxidation of MCC. All chemicals were of laboratory grade (Sigma-Aldrich, China) and used without further purification.

\subsection{Nanocellulose isolation}

\subsubsection{Acid hydrolysis (AH)}

About $5 \mathrm{~g}$ MCC was mixed with $45 \mathrm{~mL}$ sulfuric acid $(64 \mathrm{wt} \%)$, the mixture was hydrolyzed at $45^{\circ} \mathrm{C}$ for 120 min with continuous stirring (500 rpm). The hydrolysis was quenched by adding $500 \mathrm{~mL}$ water to the reaction mixture and then the slurry was washed with distilled water for $20 \mathrm{~min}$ at $5000 \mathrm{rpm}$, using repeated centrifugation. The supernatant was removed from the sediment and replaced by new distilled water and mixed, the centrifugation step continued until the $\mathrm{pH}$ of the supernatant became 1 . The last wash was conducted using dialysis with distilled water until the wash water maintained a constant $\mathrm{pH}$ of 7 . The resultant suspension was stored at $4^{\circ} \mathrm{C}$ before further analysis or treatments.

\subsubsection{TEMPO-mediated oxidation (TMO)}

About $5 \mathrm{~g}$ MCC was suspended in $500 \mathrm{~mL}$ distilled water containing TEMPO $(0.080 \mathrm{~g}, 0.5 \mathrm{mmol})$ and $\mathrm{NaBr}(0.5 \mathrm{~g}, 5 \mathrm{mmol})$. The TEMPO-mediated oxidation was started by adding $6 \% \mathrm{NaClO}$ solution $(25.0 \mathrm{mmol})$ with continuous stirring $(500 \mathrm{rpm})$ at room temperature. The $\mathrm{pH}$ was maintained at 10 by adding $0.5 \mathrm{M} \mathrm{NaOH}$ using a pH stat until no $\mathrm{NaOH}$ consumption was observed. The TEMPO-oxidized cellulose thus obtained was then ultrasonicated for $20 \mathrm{~min}$, using an ultrasonic homogenizer (KBS1200 , China) with an output power of $1200 \mathrm{~W}$. The slurry was washed with distilled water by repeated centrifugation (5000 rpm, $20 \mathrm{~min}$ ) and then centrifuged at $12000 \mathrm{rpm}$ to separate large particles. After that, the samples were dialyzed against distilled water until the $\mathrm{pH}$ reached 7 . The resultant suspension was stored at $4^{\circ} \mathrm{C}$ before further analysis or treatments.

\subsubsection{Ultrasonication (US)}

About $5 \mathrm{~g}$ MCC was dispersed in $500 \mathrm{~mL}$ distilled water under continuous stirring for a whole day, then ultrasonicated for 60 min using an ultrasonic homogenizer (KBS-1200, China) with an output 
power of $1200 \mathrm{~W}$, equipped with a sonication probe of $20 \mathrm{~mm}$. In order to avoid overheating, the beaker with the cellulose suspension was put in an ice bath with controlled temperature. Two hours after the ultrasonication process ended, nanocellulose was obtained from the water suspension by decanting the supernatant into other vessels. The resultant suspension with $\mathrm{pH}$ of 7 was stored at $4^{\circ} \mathrm{C}$ before further analysis or treatments.

\subsection{Nanocomposite films preparation}

PVA water solution $(10 \mathrm{wt} \%)$ and nanocellulose suspension ( $2 \mathrm{wt} \%$, relative to the PVA mass) were mixed under continuous stirring $(500 \mathrm{rpm})$ at $80^{\circ} \mathrm{C}$ for 3 hours, then dispersed by ultrasonic treatment (KBS-1200, China) for about 2 min with $50 \%$ power level. Films were cast onto a PTFE plate with controlled leveling, the mixture in the plate were degassed in a vacuum desiccator, and then evaporated at $25^{\circ} \mathrm{C}$ and relative humidity of $30 \%$ until films were formed, the films were heat treated in an oven at $80^{\circ} \mathrm{C}$ for more than $12 \mathrm{~h}$. Films with three levels of nanocellulose loading (2, 6 and $10 \mathrm{wt} \%$ ) were manufactured. The thicknesses of the films were controlled to be approximately $150 \mu \mathrm{m}$.

\subsection{Characterization}

\subsubsection{Morphology and crystallinity analysis}

Morphology of the nanocellulose and fractured surface of the PVA nanocomposite films were examined using scanning electron microscopy (Nova, NanoSEM 430, FEI Company) with an accelerating voltage of $15 \mathrm{kV}$. For nanocellulose observation, a droplet of the nanocellulose suspensions $(0.1 \mathrm{wt} \%)$ was put on a glass grid and dried under vacuum before SEM analysis. The dried nanocellulose and the fractured surface of the films (after tensile tests) were coated with gold on an ion sputter coater, various magnification levels were used to obtain images. More than three images were taken and chosen to observe the morphology of all samples.

Wide-angle X-ray diffraction (WXRD) data was collected using a X-ray diffractometer (Rigaku D/ max-III A, Japan) equipped with $\mathrm{Cu} \mathrm{K}_{\alpha}$ radiation ( $\lambda=$ $0.1541 \mathrm{~nm}$ ) at the operating voltage and current of $45 \mathrm{kV}$ and $100 \mathrm{~mA}$, respectively. Diffractograms were collected in a $2 \theta$ range of $4 \sim 50^{\circ}$ at a rate of $1 \%$ min with a resolution of $0.05^{\circ}$.

\subsubsection{Wet particle size and surface charge analysis}

Nanocellulose particle size analysis was conducted by dynamic light scattering (DLS) using a Zetasizer NanoZS instrument (Malvern, UK), under the following conditions: dispersant water, material refractive index 1.47, dispersion refractive index 1.33, viscosity $0.8872 \mathrm{cP}$, temperature $25^{\circ} \mathrm{C}$ and general calculation model for irregular particles. Three measurements of $10 \mathrm{~s}$ each were taken and the averaging was done.

The zeta-potential (estimated as surface charge) tests of the nanocellulose particles were conducted with the Zetasizer NanoZS Instrument (Malvern, UK). Experiments were performed in a cuvette consisting of $4 \mathrm{ml} 0.1 \mathrm{wt} \%$ nanocellulose suspension, solutions were all adjusted at $\mathrm{pH}$ values of 7 .

\subsubsection{Thermal properties}

The differential scanning calorimetry (DSC) of PVA nanocomposite films was performed on a DSC Q200 (TA Instruments, USA) from 25 to $300^{\circ} \mathrm{C}$ at a heating rate of $10^{\circ} \mathrm{C} / \mathrm{min}$ under nitrogen flow. Approximately $8 \mathrm{mg}$ samples were used. The thermogravimetric analysis (TGA) were performed using a TGA Q500 (TA Instruments, USA). About 5 10 mg samples were heated from 30 to $600^{\circ} \mathrm{C}$ with a heating rate of $10^{\circ} \mathrm{C} / \mathrm{min}$ under a nitrogen atmosphere. The weight change was recorded as a function of the heating temperature.

\subsubsection{Mechanical properties}

The mechanical tests were performed using a testing machine (Instron 5567, USA) with a crosshead speed of $5 \mathrm{~mm} / \mathrm{min}$. The crosshead extensions were used as specimen deformations. The films were cut to dog bone shapes with width of $15 \mathrm{~mm}$ for the narrow portion and total length of $50 \mathrm{~mm}$ (gauge length was $25 \mathrm{~mm}$ ). The thickness of the films was calculated before the test. The values of tensile modulus, tensile strength and elongation at break of the samples were evaluated and reported as the average values of five measurements of each composition. Prior to testing, films were kept in a humidity chamber desiccator with a $50 \%$ relative humidity $(\mathrm{RH})$ and $25^{\circ} \mathrm{C}$ for 5 days (according to the ASTM D1708 standard [20]). 


\section{Results and discussion}

\subsection{Morphological properties}

The SEM morphology of untreated MCC and nanocellulose (isolated from acid hydrolysis (AH), TEMPO-mediated oxidation (TMO) or ultrasonication (US) are shown in Figure 1. Nanocelluloses isolated from $\mathrm{AH}$ technique have individual crystallites and disperse uniformly showing needle shaped structures (namely nanocrystals), with diameters of $30 \sim 40 \mathrm{~nm}$ and lengths of $200 \sim 400 \mathrm{~nm}$. While the TMO-derived nanocellulose are interconnected webs with tiny nanofibers (namely nanofibrils), with diameters of 40 80 $\mathrm{nm}$ and the lengths are ranging from $200 \mathrm{~nm}$ to several micrometers, which have a wide range of aspect ratio, most are more than 50 as evident from the measurements of randomly selected features, nanofibrils of this aspect ratio are suitable for polymer reinforcement, in order to allow sufficient stress transfer, and thereby act as an effective reinforcing agent [21]. However, in the case of samples prepared with the US treatment, pieces of

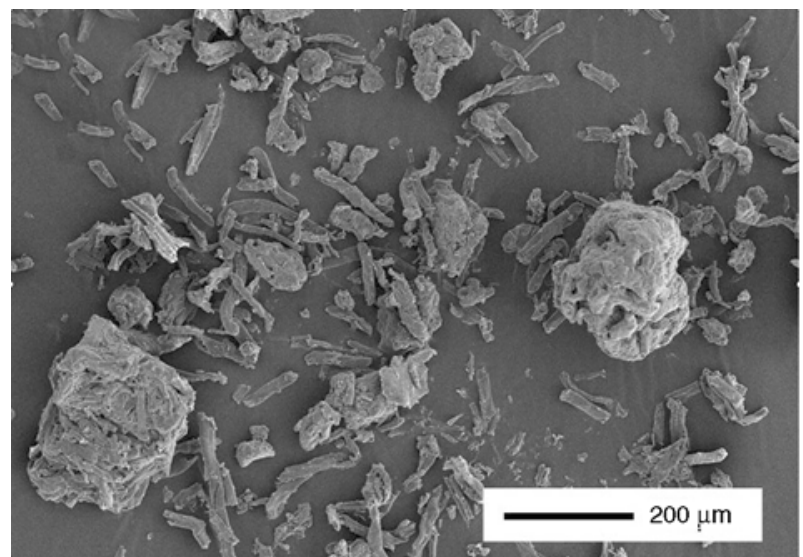

a)

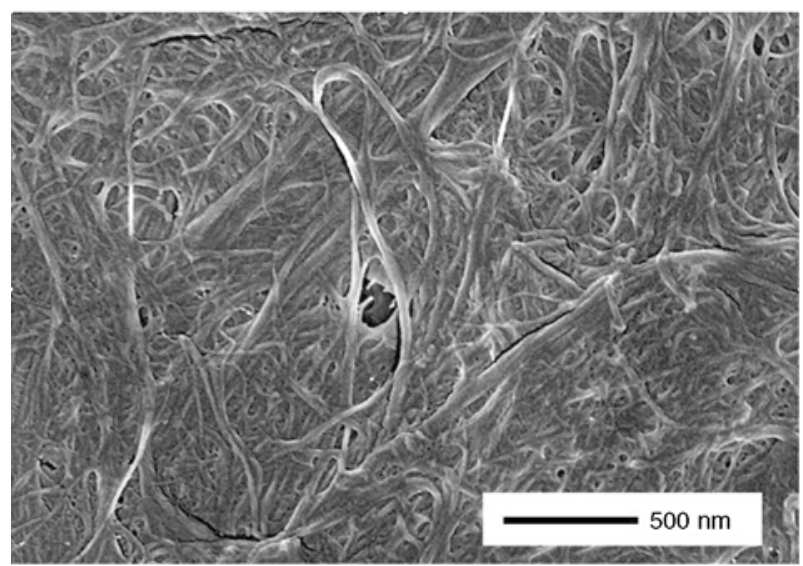

c) undefibrilated MCC and many microfibril bundles are observed.

\subsection{XRD analysis}

Figure 2 shows the X-ray diffraction of the MCC and nanocellulose (isolated from AH, TMO or US). In all curves, peaks are observed at $2 \theta=14.8,16.6$ and $22.9^{\circ}$, corresponding to a cellulose I structure,

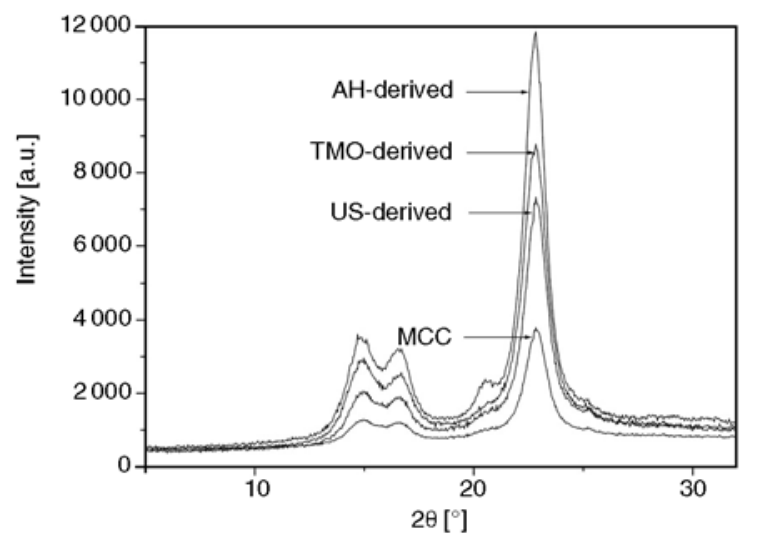

Figure 2. X-ray diffraction patterns of MCC and nanocellulose (isolated from different treatments)

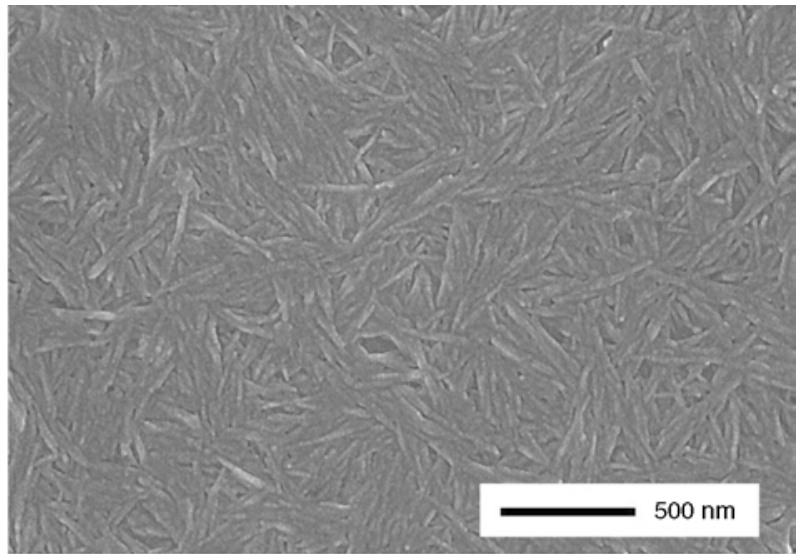

b)

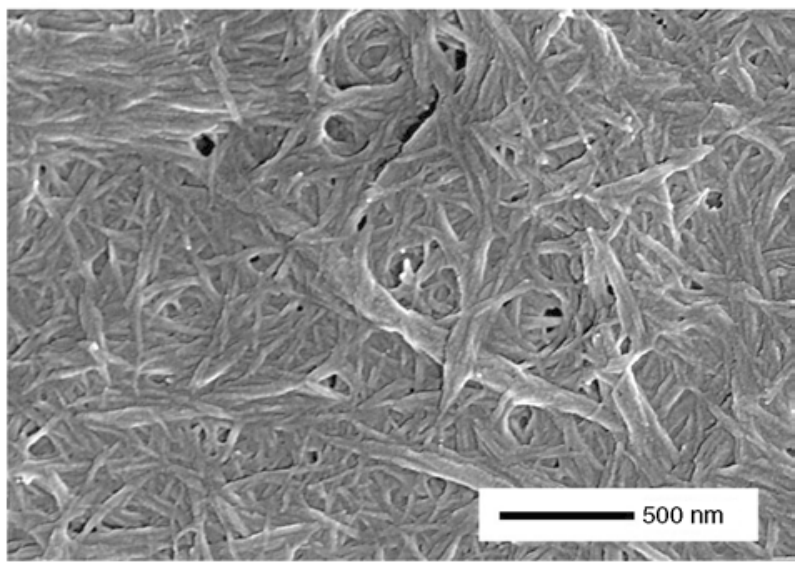

d)

Figure 1. SEM images of (a) untreated MCC, (b) AH-derived nanocellulose, (c) TMO-derived nanocellulose and (d) USderived nanocellulose. (mag 100× for (a), (mag 100000× for (b), (c), (d)) 
Table 1. Parameters of MCC and nanocellulose (isolated from different treatments)

\begin{tabular}{|l|c|c|c|c|}
\hline \multicolumn{1}{|c|}{ Nanocellulose } & MCC & AH-derived & TMO-derived & US-derived \\
\hline$X_{\mathrm{c}}[\%]$ & $79.5 \pm 2.0$ & $88.1 \pm 1.8$ & $86.4 \pm 2.0$ & $86.5 \pm 2.1$ \\
\hline Yield [\%] & - & $28.6 \pm 8.0$ & $37.4 \pm 8.3$ & $12.7 \pm 3.2$ \\
\hline Zeta [mV] & $-8.3 \pm 3.4$ & $-38.2 \pm 3.4$ & $-46.5 \pm 3.4$ & $-23.1 \pm 3.4$ \\
\hline Particle size $[\mathrm{nm}]$ & $\sim \mu \mathrm{m}$ & $115 \pm 35$ & $210 \pm 54$ & $623 \pm 93$ \\
\hline
\end{tabular}

Results expressed as mean \pm standard deviation

which means that all the three techniques have no effect on the crystal form of the native cellulose. The intensity of the peaks is higher for all the nanocellulose samples, showing that nanocellulose samples are more crystalline than MCC. The crystallinity index $\left(X_{\mathrm{c}}\right)$ of the cellulose can be calculated using the Equation (1) [22]:

$X_{\mathrm{c}}=\frac{I_{\text {crystalline }}-I_{\text {amorphous }}}{I_{\text {crystalline }}} \cdot 100$

where $I_{\text {crystalline }}$ is the intensity of the peak at $2 \theta$ about $22.9^{\circ}$ representing crystalline material and $I_{\text {amorphous }}$ is the intensity of the peak at $2 \theta$ about $18^{\circ}$ representing amorphous material in cellulosic fibers. The values of the crystallinity index obtained are shown in Table 1. The cellulose $X_{\mathrm{c}}$ is of the order AH-derived $>$ TMO-derived $>$ US-derived $>$ MCC. The maximum $X_{\mathrm{c}}(88.1 \%)$ was obtained when $\mathrm{AH}$ process was carried out for the treatment of MCC, due to the removal of the majority of amorphous regions during the harsh process and resulted in needle shaped individual crystallites. On the other hand, there is an increase of diffraction intensities in the crystalline peak around $2 \theta=20.5^{\circ}$ of AH-derived nanocellulose, which may be attributed to the higherordered region of cellulose chains. For TMO and US treatments, the nanocellulose $X_{\mathrm{c}}$ values are also high ( 86.4 and $86.5 \%$, respectively), it has been reported that TMO process produces no change in crystallinity of cellulose even at a high oxidation level of $10 \mathrm{mmol} \mathrm{NaClO} / \mathrm{g}$ cellulose [23]. The $X_{\mathrm{c}}$ increase of the TMO-derived nanocellulose in this study may be attributed to the partial removal of amorphous regions, due to the harsh ultrasonication treatment of oxidized samples.

\subsection{Yields analysis}

The yield results of the nanocellulose (isolated from $\mathrm{AH}, \mathrm{TMO}$ or US) are shown in Table 1. In cellulosic plant fibers, cellulose is present in an amorphous state, but also associates to crystalline domains through both inter-molecular and intra-molecular hydrogen bonding [24]. AH is a well-known harsh process conducted to the disintegration of amorphous regions and degradation of crystalline parts generating a low yield of $28.6 \%$. This kind of procedure affects the total integrity of fibers. While for US method, the mechanical treatment alone cannot be effective to separate the strong hydrogen bonding of native fibers. This is why the yield of USderived nanocellulose is as low as $12.7 \%$ and pieces of undefibrilated MCC still remained. While TMO is a method of combination of chemical and mechanical treatments, the mild reaction condition of room temperature, alkalescent medium and characteristic of regioselective oxidation [18-19] maintain partially amorphous regions left in the axial direction of the starting material, which result in a higher yield of $37.4 \%$, as well as higher aspect ratio of the final TMO-derived nanofibrils.

\subsection{Surface charge analysis}

Zeta potential (estimated as surface charge) can be measured by tracking the moving rate of negatively or positively charged particles across an electric field. Usually a value less than $-15 \mathrm{~m} \mathrm{~V}$ represents the onset of agglomeration. Values greater than $-30 \mathrm{mV}$ generally signifies that there is sufficient mutual repulsion which results in colloidal stability [25]. The zeta potential data of the nanocellulose suspension (isolated from $\mathrm{AH}, \mathrm{TMO}$ or US) are shown in Table 1, attributed to the esterification of cellulose hydroxyl groups to sulfonate groups during the $\mathrm{AH}$ process and the regioselective conversion of the cellulose primary hydroxyl groups to carboxyl ones during the TMO process, the AH and TMO-derived nanocellulose possess high negative charge of -38.2 and $-46.5 \mathrm{mV}$, respectively. For TMO-derived nanofibrils, the highest surface charge imparts electrostatic repulsive forces to the system, preventing the binding between nanofibrils-nanofibrils, and thus homogeneous nanocellulose suspension is obtained. The uniform dispersion of nanocellulose is critical to improve the mechanical properties of the final nano- 
composite products, promoting the actual formation of hydrogen bonding between the PVA and nanocellulose (nanocrystals or nanofibrils), which also leads to a higher efficiency of the stress transfer from the matrices to the fibers. In contrast, the US generated nanocellulose has only a weak charge of $-23.1 \mathrm{mV}$ originated from its inherent hydroxyl groups, the formation of larger insoluble precipitates was revealed by SEM image (Figure 1).
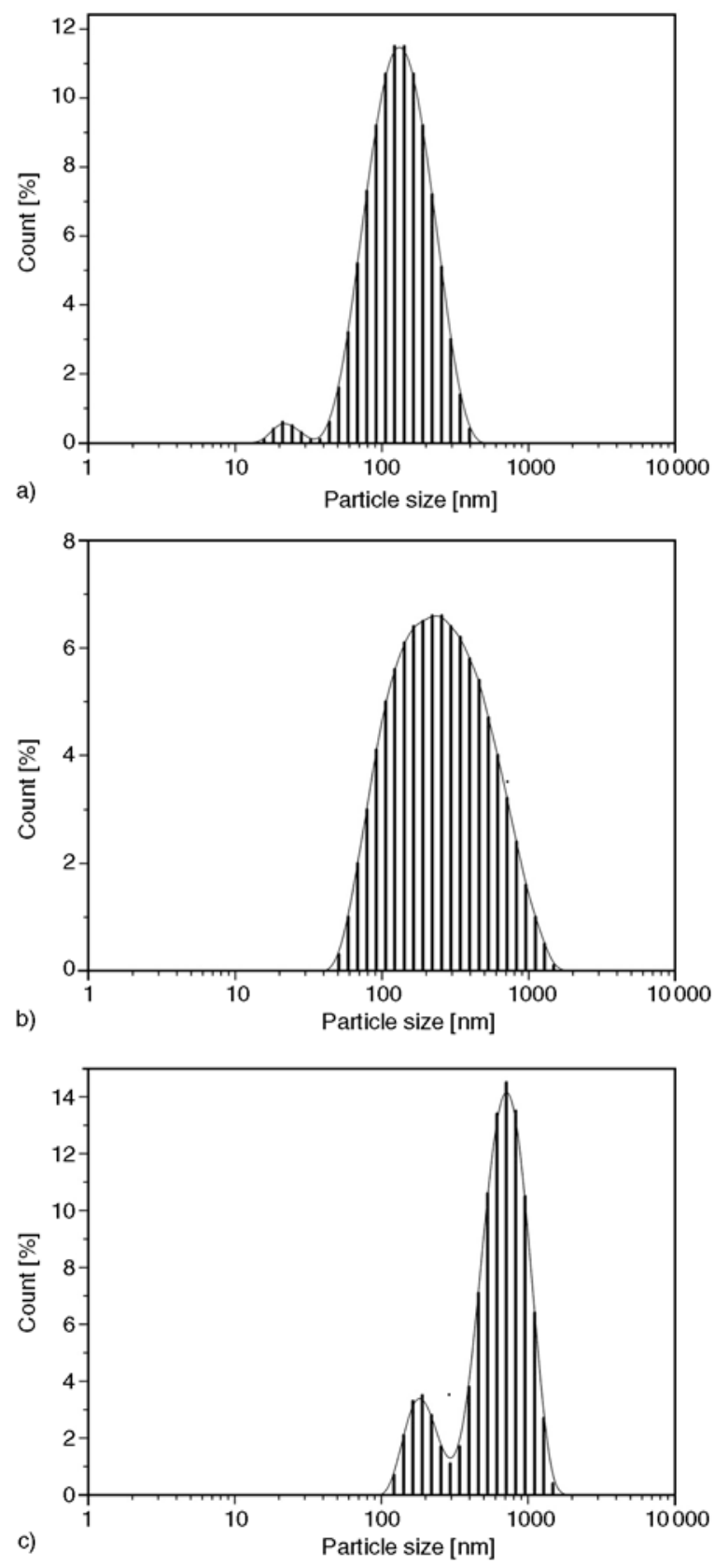

Figure 3. Size dispersion of (a) AH-derived nanocellulose, (b) TMO-derived nanocellulose, (c) US-derived nanocellulose

\subsection{Size dispersion analysis}

Dynamic light scattering (DLS) analysis has been employed to find the statistical distribution of the particles present in nanocellulose. After measuring millions of particles, an average particle size of the nanocellulose (isolated from AH, TMO or US) were found to be 115, 210 and $623 \mathrm{~nm}$ (Table 1), respectively. Larger particle size than those determined for samples in SEM analysis were obtained in DLS measurements, because of the rapid aggregation of nanocellulose in water suspension. The size dispersion of the different nanocellulose particles are shown in Figure 3. Because of the concentrated acid (64 wt $\% \mathrm{H}_{2} \mathrm{SO}_{4}$ ) hydrolysis used to harshly destroy the majority of cellulose hydrogen bonding, the $\mathrm{AH}$ reaction is homogeneous and more complete, that resulted in a narrower nanocellulose size distribution, besides, the strong surface charge $(-38.2 \mathrm{mV})$ also prevents the nanocellulose particles from agglomerating. The TMO-derived nanocellulose particles display a relatively poor size dispersion, mainly due to the mild reaction condition compared to the AH treatment. For US treatment, the lack of surface charge $(-23.1 \mathrm{mV})$ induced continuing agglomerate of insoluble nanocellulose particles, resulted in a wide size dispersion.

\subsection{Thermal properties}

Thermal characterization of neat PVA and PVA nanocomposite films was carried out using DSC and TGA measurements. From the analysis of DSC traces (Figure 4), the glass rubber transition temperature $\left(T_{\mathrm{g}}\right)$, the melting temperature $\left(T_{\mathrm{m}}\right)$, heat of

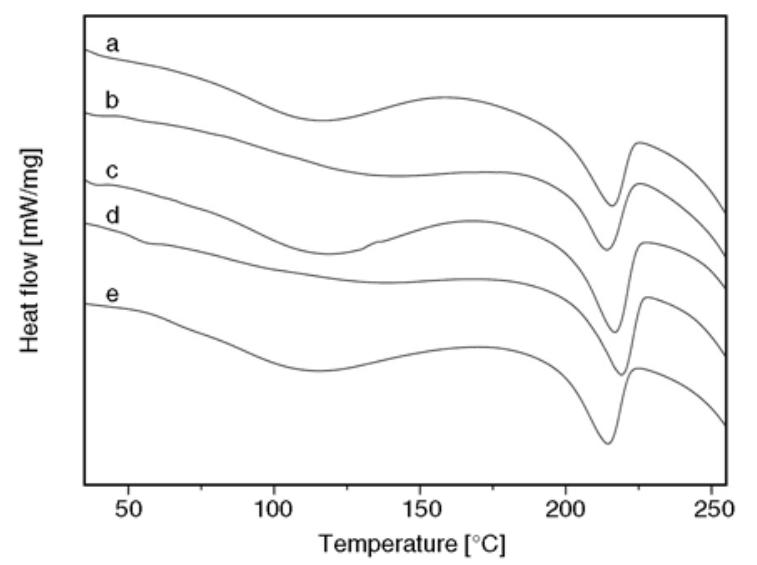

Figure 4. DSC curves of (a) neat PVA, (b) MCC/PVA, (c) AH/PVA, (d) TMO/PVA and (e) US/PVA films (6 wt $\%$ filler loading) 
Table 2. TGA data of neat PVA and PVA nanocomposite films (6 wt $\%$ filler loading)

\begin{tabular}{|l|c|c|c|c|c|}
\hline \multicolumn{1}{|c|}{ Samples } & Neat PVA & MCC/PVA & AH/PVA & TMO/PVA & US/PVA \\
\hline$T_{\mathrm{g}}\left[{ }^{\circ} \mathrm{C}\right]$ & 70.3 & 68.0 & 75.1 & 76.9 & 71.2 \\
\hline$T_{\mathrm{m}}\left[{ }^{\circ} \mathrm{C}\right]$ & 215.9 & 214.1 & 218.8 & 219.1 & 214.5 \\
\hline$\Delta H_{\mathrm{m}}[\mathrm{J} / \mathrm{g}]$ & 81.1 & 76.8 & 88.5 & 84.9 & 50.6 \\
\hline$X_{\mathrm{c}}[\%]$ & 54.1 & 54.5 & 62.8 & 60.2 & 24.1 \\
\hline$T_{10}\left[{ }^{\circ} \mathrm{C}\right]$ & 239.3 & 239.2 & 252.8 & 254.9 & 300.4 \\
\hline$T_{50}\left[{ }^{\circ} \mathrm{C}\right]$ & 283.4 & 304.5 & 306.8 & 314.9 & 264.0 \\
\hline$T_{\mathrm{d}}\left[{ }^{\circ} \mathrm{C}\right]$ & 270.4 & 271.1 & 277.2 & 287.8 & \\
\hline
\end{tabular}

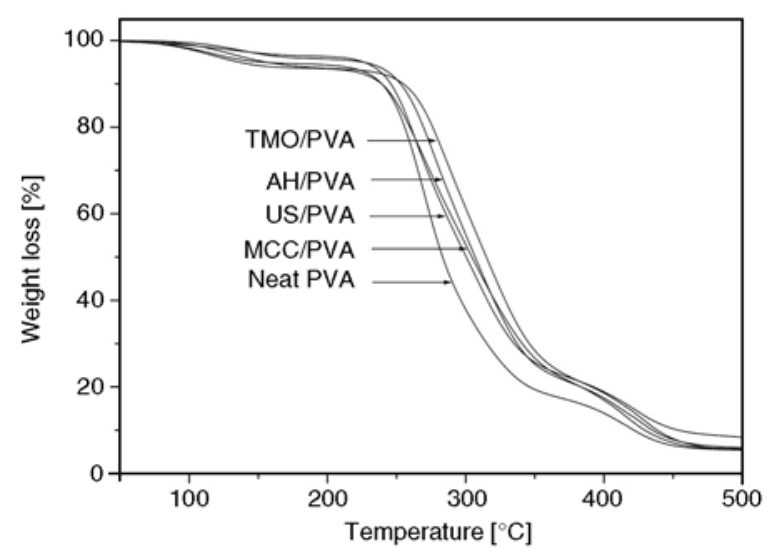

Figure 5. TGA curves of neat PVA and PVA nanocomposite films (6 $\mathrm{wt} \%$ filler loading)

fusion $\left(\Delta H_{\mathrm{m}}\right)$ and degree of crystallinity $\left(X_{\mathrm{c}}\right)$ were evaluated and compared. The resulting experimental data are listed in Table 2. The glass transition temperature $\left(T_{\mathrm{g}}\right)$ of PVA nanocomposites is increased with the addition of AH-derived and TMO-derived nanocellulose. The strong hydrogen bonding formation between the PVA matrices and nanocellulose is expected to restrict the segmental mobility of polymer chains and thereby increase $T_{\mathrm{g}}$. The features of Tm, $\Delta H_{\mathrm{m}}$, and $X_{\mathrm{c}}$ are also enhanced as compared to neat PVA films. The increase in $X_{\mathrm{c}}$ is possibly due to the nucleating effect of the nano-sized fibers. This enhancement of the Xc of the PVA matrices probably results, at least partially, in the improvement of the mechanical properties for $\mathrm{TMO} /$ PVA films as reported later. On the contrary, the thermal behavior is not obviously enhanced for US/PVA films and even tends to decrease upon $\mathrm{MCC}$ addition. It means that the presence of large agglomerate and microfibril bundles induce steric hindrance effects restricting the growth of crystalline PVA regions. It results in both a lower melting point and lower degree of crystallinity.

The thermal stability of the neat PVA and PVA nanocomposite films examined by TGA are shown in Figure 5. All samples show major weight loss in the range of $30 \sim 550^{\circ} \mathrm{C}$. For PVA nanocomposite films, a slight increase of the major degradation temperatures $\left(T_{10^{\circ} \mathrm{C}}, T_{50^{\circ} \mathrm{C}}\right.$ and $\left.T_{\mathrm{d}}\right)$ (Table 2$)$ is observed, in the sequence of TMO/PVA $>$ AH/PVA $>\mathrm{US} / \mathrm{PVA}>\mathrm{MCC} / \mathrm{PVA}>$ neat $\mathrm{PVA}$, thus further confirming the enhanced thermal stability due to a strong hydrogen bonding between the TMO-derived nanocellulose and the PVA matrices. The reason maybe that despite the regioselective oxidation of the cellulose primary hydroxyl groups to carboxylate ones in TMO treatments, the total amount of the strong hydrogen bonding in film formation is not affected. In contrast, the hydrogen bonding between the AH-derived nanocellulose and PVA matrices is decreased, due to the esterification of hydroxyl groups to sulfonate groups during the $\mathrm{AH}$ process. The US/PVA nanocomposite films have relatively lower degradation temperature. Because the specific surface area of US-derived nanocellulose is not as high as the AH-derived or TMOderived ones, resulting in fewer hydroxyl groups on the nanocellulose surface, besides, the easy formation of larger insoluble precipitates will also accelerate this process, these two factors resulted in lower thermal stability of the final composites.

\subsection{SEM and mechanical properties}

The fractured surface after tensile tests of neat PVA and PVA nanocomposite films were examined using SEM as shown in Figure 6. As compared to the neat PVA films, the morphology of the AH/PVA and TMO/PVA films can be easily identified. The nanocellulose appears as white dots, these white dots could correspond to the nanocrystals (AH-derived) or nanofibrils (TMO-derived). No large aggregates and a homogeneous distribution of the nanocellulose in the PVA matrix are observed in both AH/PVA and TMO/PVA films, implying good adhesion between fillers and matrix. This should be attributed to the formation of a rigid hydrogen-bonded 


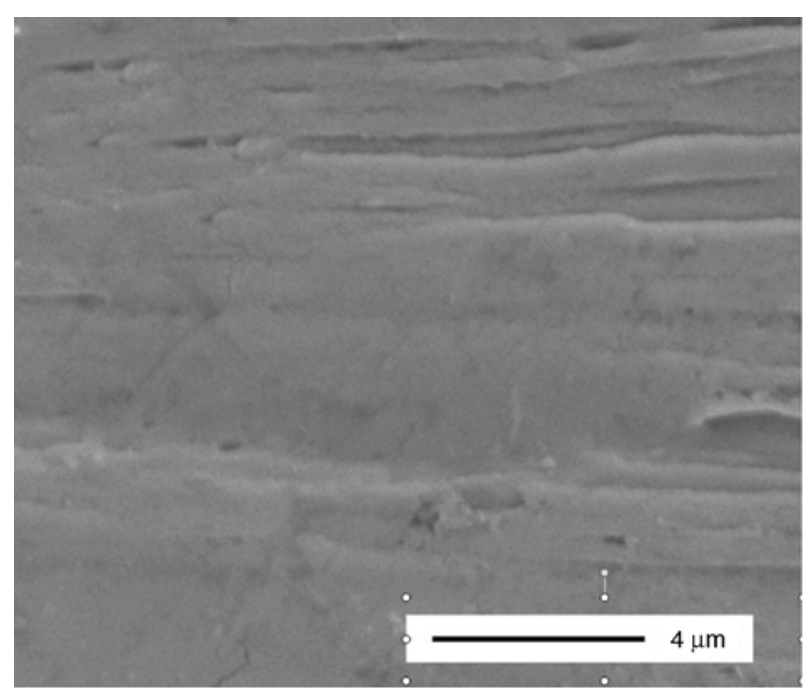

a)

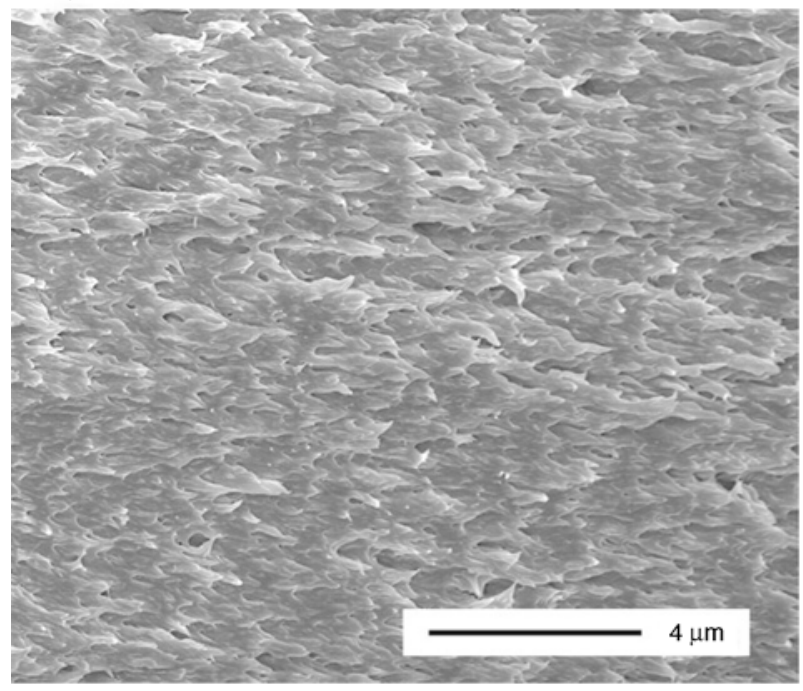

c)

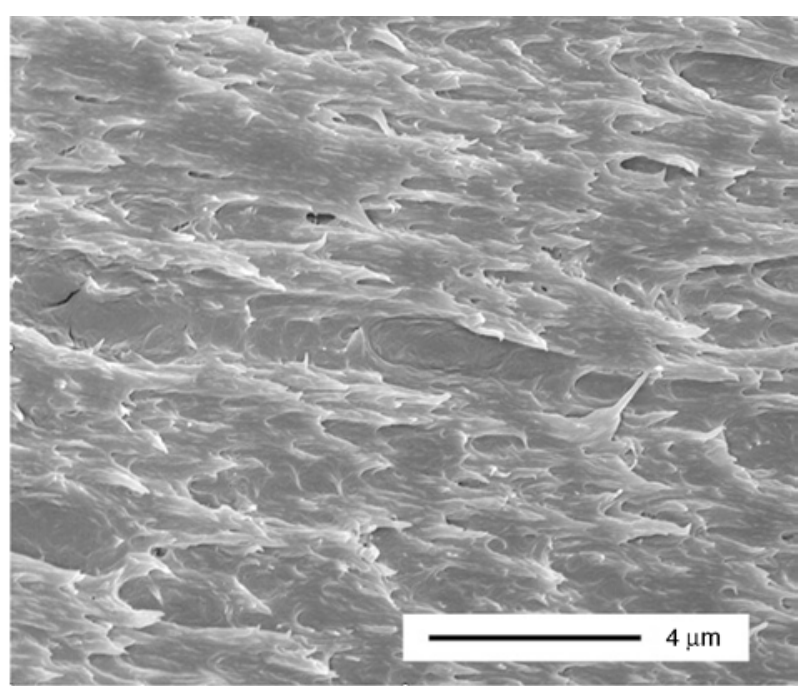

b)

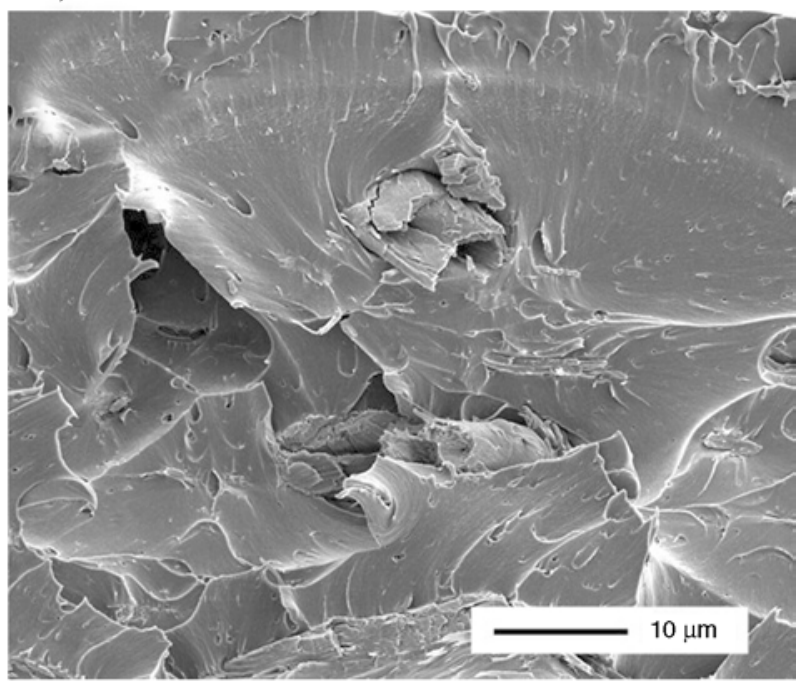

d)

Figure 6. SEM images of fractured surface of (a) neat PVA, (b) AH/PVA, (c) TMO/PVA and (d) US/PVA films.(mag $20000 \times$ for (a), (b), (c), (mag $10000 \times$ for $(d))$

network of nanocellulose that is governed by percolation theory. Such an even and uniform distribution of the fillers in the matrix could play an important role in improving the mechanical performance of the resulting nanocomposite films as discussed later. However, some microfibril bundles are observed in fractured surface of US/PVA films, because of lack of bulk charge on the US-derived nanofibrils, which makes the nanofibrils to contact and interact extensively with each other leading to the formation of loose and bulky aggregates in PVA matrices.

The tensile modulus, tensile strength and elongation at break of neat PVA and PVA nanocomposite films (2, 6, and $10 \mathrm{wt} \%$ loading) are shown in Figures 7, 8 and 9, respectively. It is found that the modulus and strength are both higher for TMO/PVA nanocomposite films, with increments of 21.5 and
$10.2 \%$ at the $6 \mathrm{wt} \%$ nanofibrils loading, respectively. The higher aspect ratio and the stronger hydrogen bonding of TMO-derived nanofibrils in the PVA matrix are the main reasons to explain this phenomenon. Whereas the elongation at break of TMO/PVA films is lower for a given loading level as compared with AH/PVA nanocomposite films. The difference is most probably ascribed to the possibility of entanglements of nanofibrils contrarily to rod-like nanocrystals. The US/PVA films are better than MCC/PVA films for modulus and elongation but similar to strength, the increase in tensile modulus or elongation of US/PVA films is less than the one reinforced by AH or TMO-derived nanocellulose. The reason is that in the US-derived nanocellulose is easy to produce larger insoluble agglomerates and always have some bigger fibrils $(>1 \mu \mathrm{m})$, 


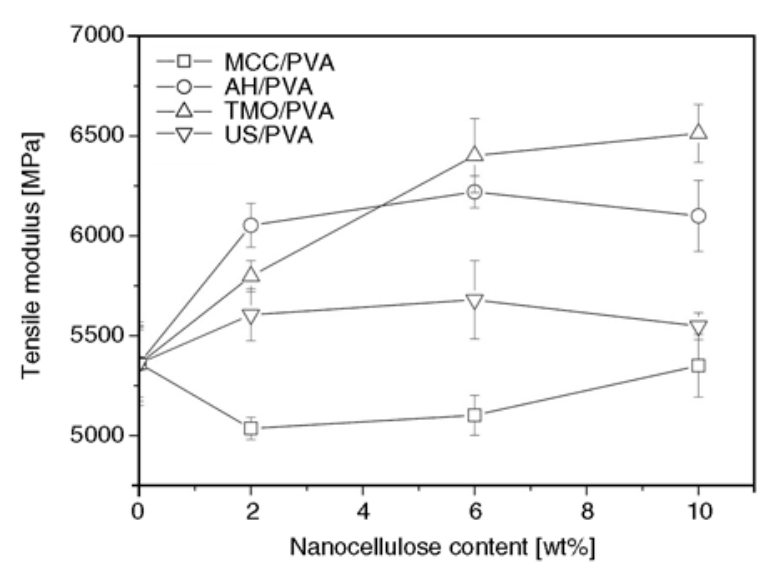

Figure 7. Tensile modulus of neat PVA and PVA nanocomposite films (2, 6 and $10 \mathrm{wt} \%$ filler loading)

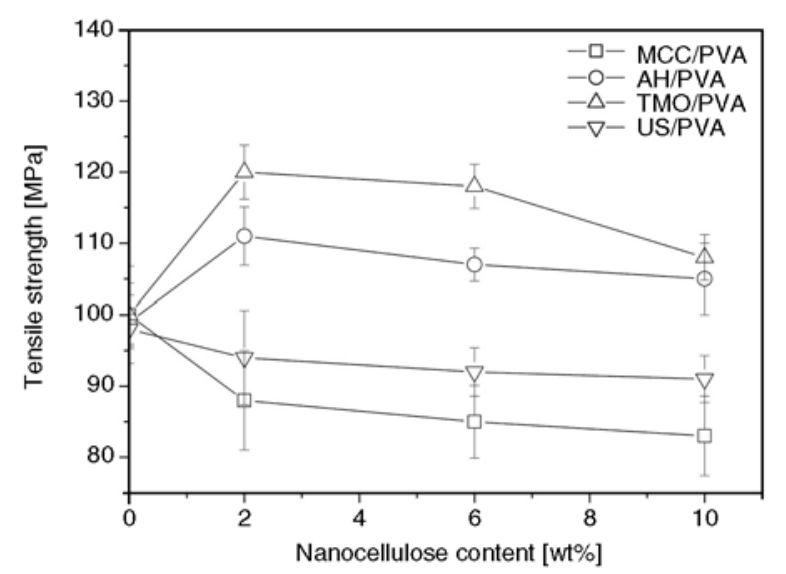

Figure 8. Tensile strength of neat PVA and PVA nanocomposite films $(2,6$, and $10 \mathrm{wt} \%$ filler loading)

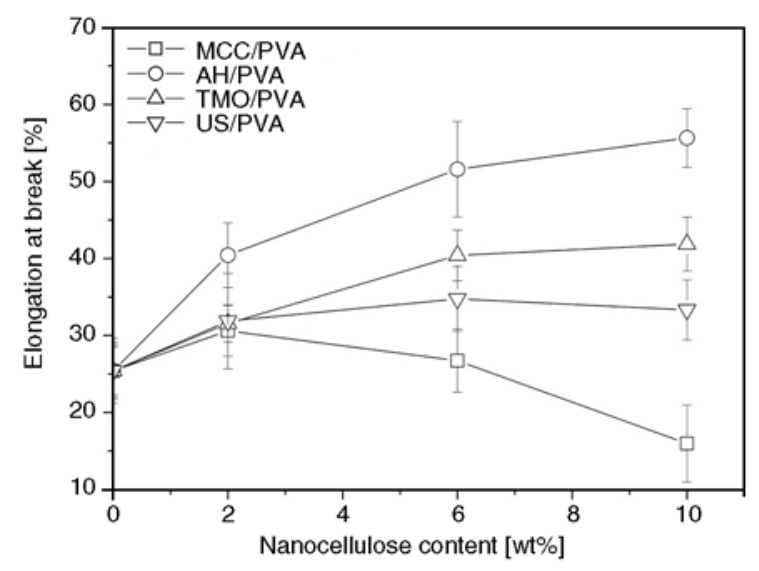

Figure 9. Elongation at break of neat PVA and PVA nanocomposite films (2, 6 and $10 \mathrm{wt} \%$ filler loading)

and because of the resulting lower aspect ratio the matrix strain exceeds that of the fibers throughout the whole length of the fibers. In such cases, the fibers do not provide efficient reinforcement, because they carry much less load than longer fibers in the same system, and hence, the composites fails before the full reinforcing potential of the fiber is attained. Besides, the fibrils may not be even and fine enough, which have more defects and low mechanical property than those of smaller nanofibrils and nanocrystal [26]. Adding more nanocellulose $(10 \mathrm{wt} \%)$ did not increase furher tensile modulus, strength or elongation, the nanocellulose agglomeration at higher concentration is the most important factor.

\section{Conclusions}

Three different techniques (acid hydrolysis (AH), TEMPO-mediated oxidation (TMO) and ultrasonication (US)) were employed to isolate nanocellulose from microcrystalline cellulose. The resulting nanocellulose was used to reinforce Poly(vinyl alcohol) (PVA) films. The characterization of nanocellulose indicated that nanocellulose with higher aspect ratio, surface charge $(-47 \mathrm{mV})$ and yields (37\%) was obtained by TMO treatment. While AH treatment resulted in higher crystallinity index (88.1\%) and better size dispersion. Both the TMOderived and AH-derived nanocellulose could homogeneous disperse in the PVA matrixes. The TMO/ PVA films were better than AH/PVA films for tensile modulus and strength but lower for elongation. The thermal behavior of the PVA nanocomposite films was higher improved with TMO-derived nanofibrils addition. Comparing to AH and US techniques, because of the mild reaction condition, the environmental friendly attribute, the good quality of resulted nanofibrils and the superior properties of the final reinforced nanocomposites the TMO technique has significant potential in the field of composites reinforcement.

\section{Acknowledgements}

This work was supported by Major State Basic Research Development Program of China (No.2010CB732206) and Doctoral Program Foundation of Institutions of Higher Education of China (20090172110022).

\section{References}

[1] Spagnol C., Rodrigues F. H. A., Pereira A. G. B., Fajardo A. R., Rubira A. F., Muniz E. C.: Superabsorbent hydrogel composite made of cellulose nanofibrils and chitosan-graft-poly(acrylic acid). Carbohydrate Polymers, 87, 2038-2045 (2012).

DOI: $\underline{10.1016 / j . c a r b p o l .2011 .10 .017}$ 
[2] Cheng Q., Wang S., Rials T.: Poly(vinyl alcohol) nanocomposites reinforced with cellulose fibrils isolated by high intensity ultrasonication. Composites Part A: Applied Science and Manufacturing, 40, 218-224 (2009).

DOI: 10.1016/j.compositesa.2008.11.009

[3] Dubief D., Samain E., Dufresne A.: Polysaccharide microcrystals reinforced amorphous poly( $\beta$-hydroxyoctanoate) nanocomposite materials. Macromolecules, 32, 5765-5771 (1999).

DOI: $10.1021 / \mathrm{ma990274a}$

[4] Eichhorn S. J., Dufresne A., Aranguren M., Marcovich N. E., Capadona J. R., Rowan S. J., Weder C., Thielemans W., Roman M., Renneckar S., Gindl W., Veigel S., Keckes J., Yano H., Abe K., Nogi M., Nakagaito A. N., Mangalam A., Simonsen J., Benight A. S., Bismarck A., Berglund L. A., Peijs T.: Review: Current international research into cellulose nanofibres and nanocomposites. Journal of Materials Science, 45, 133 (2010).

DOI: $\underline{10.1007 / \mathrm{s} 10853-009-3874-0}$

[5] Lee S-Y., Mohan D. J., Kang I-A., Doh G-H., Lee S., Han S. O.: Nanocellulose reinforced PVA composite films: Effects of acid treatment and filler loading. Fibers and Polymers, 10, 77-82 (2009).

DOI: $10.1007 / \mathrm{s} 12221-009-0077-\mathrm{x}$

[6] Abraham E., Deepa B., Pothan L. A., Jacob M., Thomas S., Cvelbar U., Anandjiwala R.: Extraction of nanocellulose fibrils from lignocellulosic fibres: A novel approach. Carbohydrate Polymers, 86, 14681475 (2011).

DOI: 10.1016/j.carbpol.2011.06.034

[7] Mandal A., Chakrabarty D.: Isolation of nanocellulose from waste sugarcane bagasse (SCB) and its characterization. Carbohydrate Polymers, 86, 1291-1299 (2011). DOI: $10.1016 /$ j.carbpol.2011.06.030

[8] Morán J. I., Alvarez V. A., Cyras V. P., Vázquez A.: Extraction of cellulose and preparation of nanocellulose from sisal fibers. Cellulose, 15, 149-159 (2008). DOI: $10.1007 / \mathrm{s} 10570-007-9145-9$

[9] Siqueira G., Tapin-Lingua S., Bras J., da Silva Perez D., Dufresne A.: Morphological investigation of nanoparticles obtained from combined mechanical shearing, and enzymatic and acid hydrolysis of sisal fibers. Cellulose, 17, 1147-1158 (2010).

DOI: $10.1007 / \mathrm{s} 10570-010-9449-\mathrm{Z}$

[10] Siqueira G., Tapin-Lingua S., Bras J., da Silva Perez D., Dufresne A.: Mechanical properties of natural rubber nanocomposites reinforced with cellulosic nanoparticles obtained from combined mechanical shearing, and enzymatic and acid hydrolysis of sisal fibers. Cellulose, 18, 57-65 (2011). DOI: $10.1007 / \mathrm{s} 10570-010-9463-1$
[11] Nickerson R. F., Habrle J. A.: Cellulose intercrystalline structure. Industrial and Engineering Chemistry Research, 39, 1507-1512 (1947).

DOI: $10.1021 / \mathrm{ie} 50455 \mathrm{a} 024$

[12] Cao X., Chen Y., Chang P. R., Muir A. D., Falk G.: Starch-based nanocomposites reinforced with flax cellulose nanocrystals. Express Polymer Letters, 2, 502510 (2008).

DOI: $10.3144 /$ expresspolymlett.2008.60

[13] Wang S., Cheng Q.: A novel process to isolate fibrils from cellulose fibers by high-intensity ultrasonication, Part 1: Process optimization. Journal of Applied Polymer Science, 113, 1270-1275 (2009).

DOI: 10.1002/app.30072

[14] Chakraborty A., Sain M., Kortschot M.: Cellulose microfibrils: A novel method of preparation using high shear refining and cryocrushing. Holzforschung, 59, 102-107 (2005).

DOI: $10.1515 / \mathrm{HF} .2005 .016$

[15] Abe K., Iwamoto S., Yano H.: Obtaining cellulose nanofibers with a uniform width of $15 \mathrm{~nm}$ from wood. Biomacromolecules, 8, 3276-3278 (2007).

DOI: $10.1021 / \mathrm{bm} 700624 \mathrm{p}$

[16] Nakagaito A. N., Yano H.: The effect of morphological changes from pulp fiber towards nano-scale fibrillated cellulose on the mechanical properties of high-strength plant fiber based composites. Applied Physics A: Materials Science and Processing, 78, 547-552 (2004). DOI: $10.1007 / \mathrm{s} 00339-003-2453-5$

[17] de Souza Lima M. M., Borsali R.: Rodlike cellulose microcrystals: Structure, properties, and applications. Macromolecular Rapid Communications, 25, 771-787 (2004).

DOI: $10.1002 /$ marc. 200300268

[18] Saito T., Kimura S., Nishiyama Y., Isogai A.: Cellulose nanofibers prepared by TEMPO-mediated oxidation of native cellulose. Biomacromolecules, 8, 2485-2491 (2007).

DOI: $10.1021 / \mathrm{bm} 0703970$

[19] Saito T., Nishiyama Y., Putaux J-L., Vignon M., Isogai A.: Homogeneous suspensions of individualized microfibrils from TEMPO-catalyzed oxidation of native cellulose. Biomacromolecules, 7, 1687-1691 (2006). DOI: $10.1021 / \mathrm{bm} 060154 \mathrm{~s}$

[20] ASTM 1708-2002a: Standard test method for tensile properties of plastics by use of microtensile specimens (2002).

[21] Cheng Q., Wang S., Rials T. G., Lee S-H.: Physical and mechanical properties of polyvinyl alcohol and polypropylene composite materials reinforced with fibril aggregates isolated from regenerated cellulose fibers. Cellulose, 14, 593-602 (2007). DOI: $10.1007 / \mathrm{s} 10570-007-9141-0$ 
[22] Thygesen A., Oddershede J., Lilholt H., Thomsen A. B., Ståhl K.: On the determination of crystallinity and cellulose content in plant fibres. Cellulose, 12, 563576 (2005).

DOI: $10.1007 / \mathrm{s} 10570-005-9001-8$

[23] Johnson R. K., Zink-Sharp A., Renneckar S. H., Glasser W. G.: A new bio-based nanocomposite: Fibrillated TEMPO-oxidized celluloses in hydroxypropylcellulose matrix. Cellulose, 16, 227-238 (2009). DOI: $10.1007 / \mathrm{s} 10570-008-9269-6$

[24] Fengel D., Wegner G.: Wood-chemistry, ultrastructure, reactions. Walter de Gruyter, Berlin (1989).
[25] Khouri S.: Experimental characterization and theoretical calculations of responsive polymeric systems. Master's thesis University of Waterloo, Waterloo, Canada (2010).

[26] Cheng Q., Wang S., Harper D. P.: Effects of process and source on elastic modulus of single cellulose fibrils evaluated by atomic force microscopy. Composites Part A: Applied Science and Manufacturing, 40, 583588 (2009).

DOI: $10.1016 /$ j.compositesa.2009.02.011 